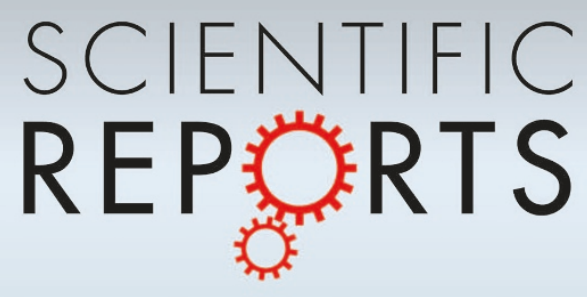

OPEN

SUBJECT AREAS:

PARKINSON'S DISEASE

CELLULAR NEUROSCIENCE

MICROGLIA

CELL DEATH IN THE NERVOUS

SYSTEM

Received

29 May 2012

Accepted

18 January 2013

Published

6 March 2013

Correspondence and requests for materials should be addressed to S.H. (stephane.huno@@ upmc.fr) or A.H. (andreas.hartmann@ psl.aphp.fr)

\title{
Toll like receptor 4 mediates cell death in a mouse MPTP model of Parkinson
} disease

\author{
Carmen Noelker ${ }^{1,2}$, Lydie Morel' ', Thomas Lescot ${ }^{1}$, Anke Osterloh ${ }^{3}$, Daniel Alvarez-Fischer ${ }^{1,2}$, \\ Minka Breloer ${ }^{3}$, Carmen Henze' ${ }^{1}$, Candan Depboylu' ${ }^{2}$, Delphine Skrzydelski' ${ }^{1}$, Patrick P. Michel' \\ Richard C. Dodel' ${ }^{2}$ Lixia Lu', Etienne C. Hirsch', Stéphane Hunot' \& Andreas Hartmann'
}

'INSERM UMR_S975, Université Pierre et Marie Curie Paris 06 UMR_S975, CNRS UMR 7225, CR-ICM, Groupe Hospitalier Pitié-Salpêtrière, 75013 Paris, France, ${ }^{2}$ Department of Neurology, Philipps-University Marburg, 35043 Marburg, Germany, ${ }^{3}$ Bernhard Nocht Institute for Tropical Medicine, Hamburg, 20324 Germany.

In mammalians, toll-like receptors (TLR) signal-transduction pathways induce the expression of a variety of immune-response genes, including inflammatory cytokines. It is therefore plausible to assume that TLRs are mediators in glial cells triggering the release of cytokines that ultimately kill DA neurons in the substantia nigra in Parkinson disease (PD). Accordingly, recent data indicate that TLR4 is up-regulated by 1-methyl-4-phenyl-1,2,3,6-tetrahydropyridine (MPTP) treatment in a mouse model of PD. Here, we wished to evaluate the role of TLR4 in the acute mouse MPTP model of PD: TLR4-deficient mice and wild-type littermates control mice were used for the acute administration way of MPTP or a corresponding volume of saline. We demonstrate that TLR4-deficient mice are less vulnerable to MPTP intoxication than wild-type mice and display a decreased number of Iba1 + and MHC II+ activated microglial cells after MPTP application, suggesting that the TLR4 pathway is involved in experimental PD.

2 arkinson disease (PD) is a common neurodegenerative disorder clinically characterized by akinesia, rigidity and rest tremor ${ }^{1}$. The cardinal neuroanatomical feature of PD is a massive and preferential loss of dopaminergic (DA) neurons in the substantia nigra pars compacta (SNpc) of patients, resulting in a drastic decline in striatal dopamine concentrations.

Another histopathological hallmark of PD is microglial activation in the $\mathrm{SNpc}{ }^{2,3}$. Activated microglia are believed to contribute to the neurodegenerative process through the release of pro-inflammatory and/or cytotoxic factors such as interleukin- $1 \beta$ (IL-1 $\beta$ ), tumor necrosis factor- $\alpha$ (TNF- $\alpha$ ), nitric oxide (NO) and reactive oxygen intermediates ${ }^{2}$. In mammalians, toll-like receptors (TLR) signal-transduction pathways induce the expression of a variety of immune-response genes, including inflammatory cytokines ${ }^{4}$. It is therefore plausible to assume that TLRs are mediators in glial cells triggering the release of cytokines that ultimately kill DA neurons in the SNpc.

The best characterized TLR transduction pathway is that of TLR4 which functions extracellularly in conjunction with CD14 and MD-2, the so-called lipopolysaccharide (LPS) recognition complex, which signals downstream through the myeloid differentiation factor 88 (MyD88) pathway. Accordingly, as first demonstration in the central nervous system (CNS), the only cellular population stained positive for TLR4 in the brain parenchyma of adult rats were microglia ${ }^{5}$. Recent data also indicate that TLR4 is up-regulated by MPTP treatment in a mouse model of $\mathrm{PD}^{6}$, may promote alpha-synuclein clearance in a model of atypical parkinsonism ${ }^{7}$ and that alphasynuclein directly activates microglia, inciting the production of proinflammatory molecules and altering the expression of TLRs ${ }^{8,9}$. Finally, MyD88 deletion protects against MPTP neurotoxicity, albeit only in the enteric and not in the central nervous system ${ }^{10,11}$.

In the light of these findings, we wished to evaluate the role of TLR4 in the acute mouse MPTP model of PD using mice deleted for TLR4 and their wild type littermates.

\section{Results}

We compared MPTP-induced nigrostriatal injury between TLR4-deficient mice and their WT littermates. We observed a significant difference between saline-treated TLR4-deficient mice and WT animals regarding basal dopamine levels (Figure 1A, $72.6 \pm 1.5 \mathrm{ng} / \mathrm{mg}$ protein vs. $89.3 \pm 3.4 \mathrm{ng} / \mathrm{mg}$ protein). However, DA metabolites 

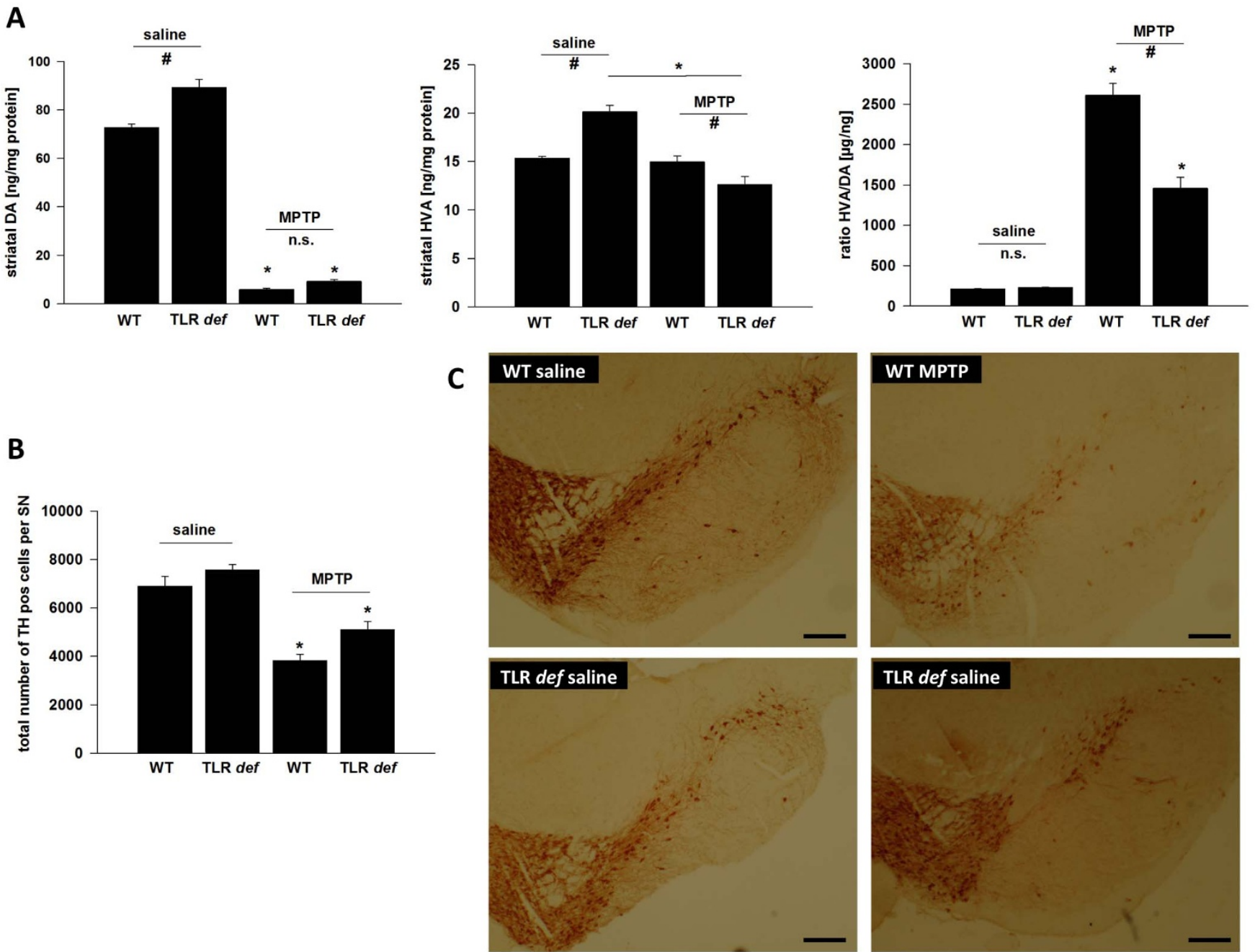

Figure $1 \mid$ (A): HPLC measurement of striatal dopamine (DA) and homovanillic acid (HVA) levels, as well as calculation of the HVA/DA-ratio after MPTP-intoxication of TLR4- deficient (TLR def) and WT mice in comparison to saline treated mice. Values are expressed as mean \pm SEM ${ }^{*} \mathrm{p}<0.05$ comparing MPTP treated group with its saline control, \#p $<0.05$ comparing MPTP-treated groups of WT and TLR4-deficient mice. (B): TH-positive cell number in SN after MPTP treatment. WT $=$ WT mice, TLR deficient $=$ TLR4 deficient mice. Values are expressed as mean \pm SEM $\left({ }^{*} \mathrm{p}<0.05\right.$ comparing MPTP treated group with its saline control). (C): Representative photographs of the SN of each treatment group is shown. $\mathrm{TH}$-staining of free-floating cryomicrotome-cut sections at day 7. TLR4-deficient $=$ TLR4-deficient mice, $\mathrm{WT}=$ wild-type mice. Control $=$ saline treated mice, MPTP $=$ MPTP-treated mice, Scale bars $=200 \mu \mathrm{m}$.

were increased in both groups under basal conditions, indicating that dopamine turnover and metabolism were identical regardless of genotype $(211.8 \pm 5.2 \mu \mathrm{g} / \mathrm{ng}$ vs. $226.0 \pm 6.8 \mu \mathrm{g} / \mathrm{ng}$, Figure 1A $)$. After MPTP treatment, striatal DA was reduced significantly in both groups; however, there was no significant difference in reduction with regard to genotype $(5.9 \pm 0.6 \mathrm{ng} / \mathrm{mg}$ vs. $9.2 \pm 0.8 \mathrm{ng} / \mathrm{mg}$, Figure 1A). The ratio of HVA/DA was significantly increased in both groups after MPTP treatment, reflecting the lesion of the nigrostriatal pathway. However, in mice lacking the TLR4 receptor increase in HVA/DA was significantly less pronounced, indicating a weaker impact of the toxin on cell function in those animals (2611.3 $\pm 145.0 \mu \mathrm{g} / \mathrm{ng}$ vs. $1458.1 \pm 138.1 \mu \mathrm{g} / \mathrm{ng}$, Figure 1A).

Accordingly, TLR4-deficient mice displayed an increased number of surviving $\mathrm{TH}+$ neurons compared to WT mice (total number of $\mathrm{TH}^{+}$- cells/SN: $6906 \pm 394(\mathrm{WT} /$ saline), $7578 \pm 430$ (TLR4 deficient/saline), $3828 \pm 523$ (WT/MPTP) und $5119 \pm 326$ (TLR4 deficient/MPTP) suggesting that TLR4-deficient mice could be partially protected against MPTP toxicity at the perykaryal level (Figs. 1B and C). However, two-way ANOVA revealed only statistical differences for genotype and treatment but failed to detect a significant interaction between both factors, most likely due to the low power of the test (power for interaction with alpha $=0.05$ ). Since the number of Nissl-positive/TH-negative neurons did not increase in MPTPtreated animals, the decrease in TH-positive neurons could be attributed to cell loss rather than loss of TH protein expression (percentage of TH-negative and Nissl-positive neurons in SNpc; TLR4-deficient mice $(97.3 \% \pm 5.2 \%)$, WT mice $(95.8 \% \pm 4.7 \%)$. Furthermore, reduced MPTP metabolism in mutant mice is not likely to account for the observed neuroprotection, as striatal $\mathrm{MPP}^{+}$content after a single systemic MPTP injection did not differ between genotypes (Supplemental Figure 1).

Finally, to establish a possible link between TLR4 function and microglial cell activation, we assessed the extent of $\mathrm{Iba}^{+}$microglial cells within the SN of MPTP-intoxicated WT and TLR4-deficient mice at day 2 and day 7 after MPTP-intoxication. As shown in Fig. 2A there were no differences observed between WT control mice and TLR4-deficient control mice (total number of Iba1 ${ }^{+}$- microglial cells/SN: 1437,5 \pm 77,24 (WT/saline), 1392,5 \pm 60,14 (TLR4 deficient/saline), but TLR4-deficient mice showed a significantly decreased number of nigral microglial cells after day 2 and day 7 (total number of $\mathrm{Ibal}^{+}$- microglial cells/SN: 5342,5 \pm 121,59 (WT, MPTP 2d), 3891,25 \pm 154,62 (TLR4 deficient, MPTP 2d); $4786 \pm$ 


\section{C}

A
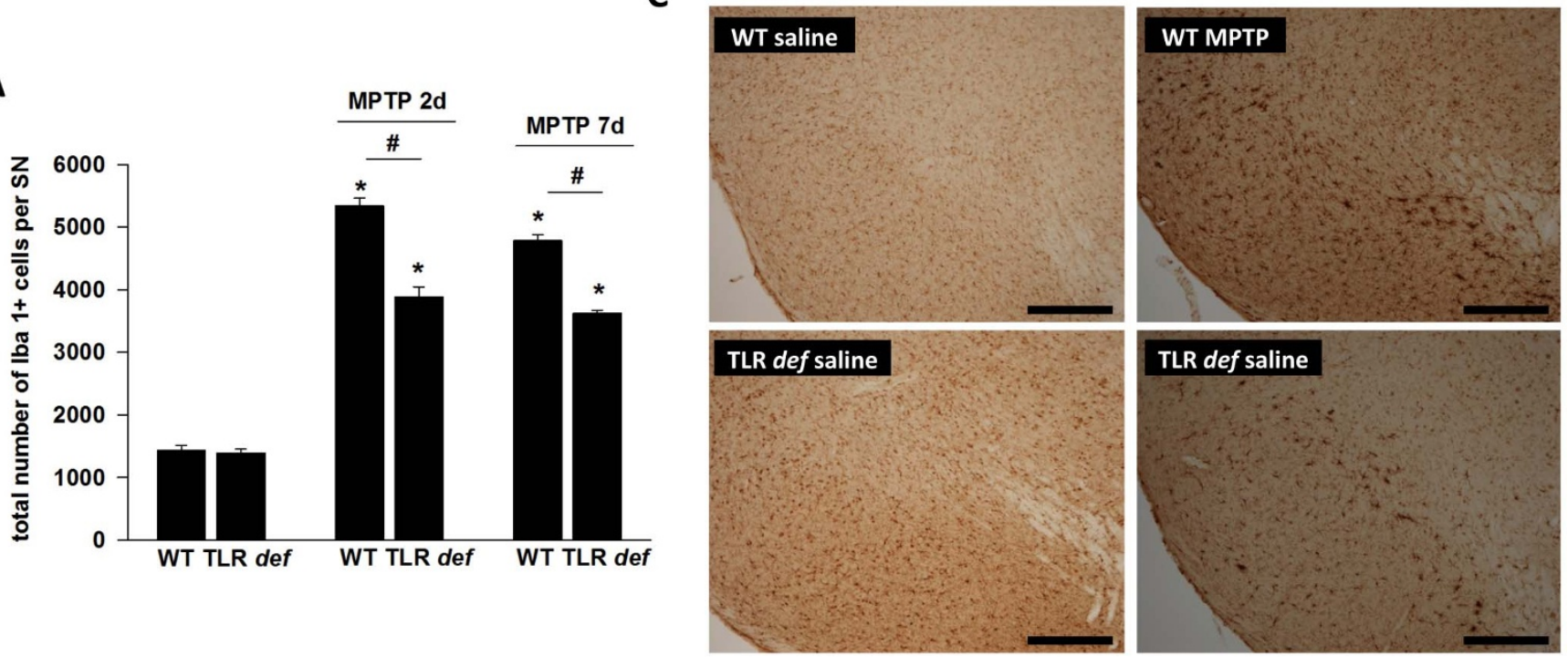

B

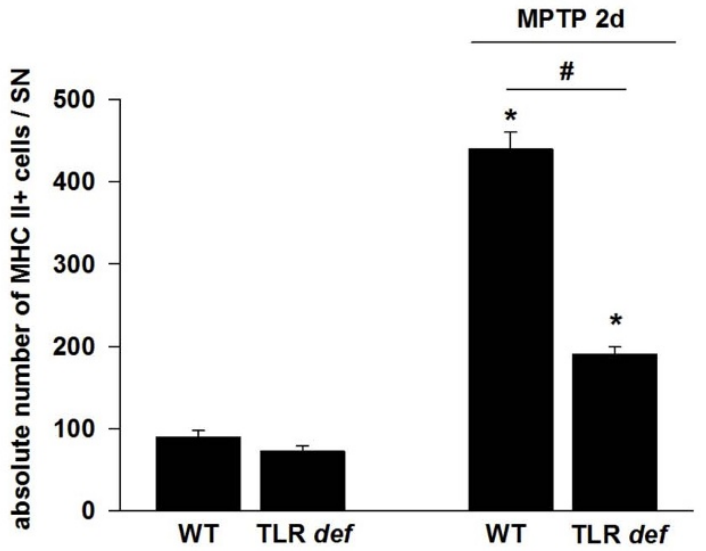

D

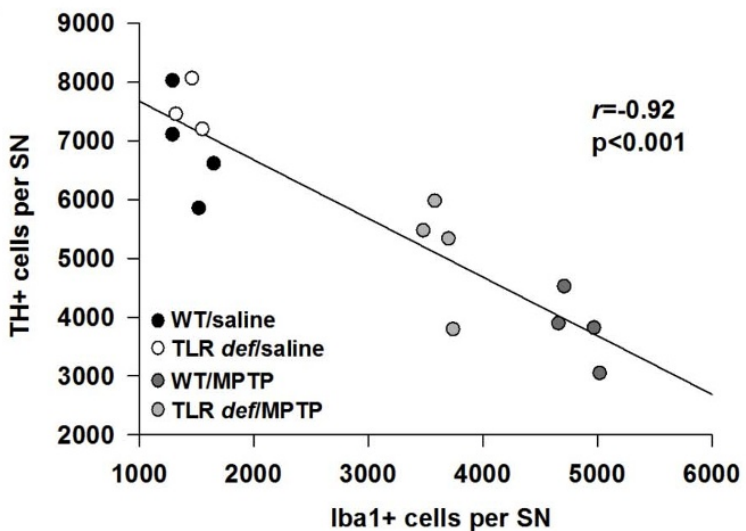

Figure $2 \mid$ (A): Number of nigral $\mathrm{Iba}^{+}$microglia at day 2 and day7. WT $=$ WT mice, TLR def $=$ TLR4-deficient mice. Values are expressed as mean \pm SEM $\left({ }^{*} \mathrm{p}<0.05\right.$ comparing MPTP-treated group with its saline control, \#p $<0.05$ comparing MPTP-treated groups of WT and TLR4-deficient mice). (B): MHC II + cell number in SN after MPTP treatment at day 2. WT $=$ WT mice, TLR def $=$ TLR4-deficient mice. Values are expressed as mean \pm SEM $\left({ }^{*} \mathrm{p}<0.05\right.$ comparing MPTP-treated group with its saline control, \#p $<0.05$ comparing MPTP-treated groups of WT and TLR4-deficient mice). (C): Representative photographs of the SN (Iba- 1 staining) of each treatment group at day 2 are shown. TLR4 def $=$ TLR4-deficient mice, WT $=$ wild-type mice. Control $=$ saline treated mice, MPTP $=$ MPTP treated mice. Scale bars $=200 \mu \mathrm{m}$. (D): Correlation analysis of TH ${ }^{+}$- and Iba ${ }^{+}$-positive cells counts at day 7 in $\mathrm{SNpc}(\mathrm{r}=-0.92, \mathrm{p}<0.001)$.

88,571,6 (WT/MPTP 7d); $3625 \pm$ 51,17 (TLR4-deficient/MPTP 7d) after MPTP intoxication. In Figure 2C representative photographs of the $\mathrm{SN}$ of each treatment group at day 2 are shown.

To demonstrate additional microglia markers we performed $\mathrm{MHC}$ II staining at day 2 (Fig. 2B) where no differences were detectable between WT and TLR4-deficient mice (total number of MHC II+ microglial cells/SN: $90 \pm$ 7,98 (WT/saline); 72,5 \pm 6,49 (TLR4 deficient/saline). Consistent with the results of the Iba1-staining, TLR4deficient mice showed a significantly decreased number of nigral activated microglial cells after day 2 compared to WT mice (total number of MHC II + microglial cells/SN: $440 \pm 20$ (WT/MPTP) und $190 \pm$ 9,51 (TLR4-deficient/MPTP).

Additionally, we performed a correlation analysis of $\mathrm{TH}^{+}$- and $\mathrm{Iba}^{+}$-positive cells counts at day 7 where a highly negative correlation coefficient could be calculated between the two variables $(r=-0.92$, $\mathrm{p}<0.001$, Fig. 2C).

\section{Discussion}

Here, we show that TLR4-deficient mice are partially protected against MPTP toxicity which correlates with decreased microglial activation in the $\mathrm{SN}$, suggesting that dopaminergic cell death in this paradigm is, at least to some extent, TLR4-dependent. Sustentative to this result, a recent study observed an augmented expression of TLR4 and CD14 in the SN of mice treated with MPTP in comparison to control animals ${ }^{20}$. More recently, our group was able to demonstrate upregulation of several TLR (including TLR4) in the MPTP-lesioned SNpc (acute protocol) ${ }^{6}$. Moreover, a case-control study showed that a genetic variations within the CD14 monocyte receptor gene $(\mathrm{C}-260 \mathrm{~T})$ was associated with increased risk of PD in females ${ }^{21}$. Both studies underline the potential implication of the TLR4/CD14 pathway in PD pathogenesis.

Drouin-Ouellet et al. ${ }^{11}$ were unable to demonstrate a protective effect of MyD88 deletion, the downstream effector pathway to TLR4, against MPTP. However, this group used a subacute MPTP protocol much less likely to induce neuroinflammation than the acute MPTP protocol used in the present study. An alternative, but less likely scenario involves signaling of TLR4 through the TIR-domain-containing adapter-inducing interferon- $\beta$ (TRIF)-dependent pathway. Specifically, the TRIF-dependent pathway is able to activate NF- $\kappa \mathrm{B}$, and additionally activates interferon regulatory factor 3 to induce 
type I IFNs. TLR4 can also activate the TRIF-dependent pathway from late endosomes following phagocytosis. However, activation of this pathway never occurs totally independently of MyD88 activation $^{4}$. Another issue concerns the use of $\mathrm{C} 3 \mathrm{H} / \mathrm{HeJ}$ mutant mice as a model for TLR4 deficiency. In addition to TLR4, a range of abnormalities have been identified in these mice by quantitative trait locus mapping on chromosomes 4, 6 and 19, which might also account for the altered basal dopamine metabolism compared to $\mathrm{C} 3 \mathrm{H} / \mathrm{OuJ}$ mice $^{22}$. These alterations could potentially confound the interpretations of the resistance of these mice to MPTP toxicity.

Interestingly, Stefanova et al. ${ }^{7}$ suggest that normal TLR4 function might be necessary to maintain the phagocytic response of microglia to alpha-synuclein, albeit in a multisystem atrophy model where alpha-synuclein is acummulating in oligodendroglia as opposed to neurons. Nonetheless, these data underly the need for caution when balancing anti- and pro-survival effects of specific receptors and pathways in the context of pathology.

In this respect, it might be postulated that PD-associated pathology reflects a sterile tissue damage (i.e. not mediated by pathogens) condition. In this scenario, brain resident macrophage-like microglia represent important innate immune cells that can be activated by endogenous molecules. Our data suggest that TLR4 is involved in this endogenous activator complex. However, the endogenous activator of TLR4 in this scenario could not be identified yet. A variety of endogenous TLR ligands, for instance heat schock proteins, might contribute significantly to sterile inflammation, and appropriate antagonists may represent a new class of therapeutic agents for such diseases $^{23,24}$. We acknowlegde that, based on our findings, the contribution of the TLR4-dependent pathway in PD may be modest. Nonetheless, a $10 \%$ difference in cell loss can mean the difference between the appearance or supression of clinical symptoms in patients with early PD. Moreover, therapeutic development in PD may not rely on a single, "magic bullet" drug but on a combination of molecules, so-called cocktails. Therefore, exploring the pieces of the puzzle, the different receptors and pathways, does seem a worthwile effort to us. Further studies will reveal whether pharmacological modulations in the TLR4-pathway could represent a novel therapeutic approach in the treatment of PD.

\section{Methods}

Animals. Animals were housed, handled, and cared for in accordance with the Guide for the Care and Use of Laboratory Animals [NCR (National research council) 1996] and the European Union Council Directive 86/609/EEC, and the experimental protocols were carried out in compliance with institutional ethical committee guidelines (Université Pierre et Marie Curie - UPMC) for animal research.

MPTP intoxication in TLR4-deficient mice. Adult (8-10 weeks) male TLR4-deficient mice and wildtype (WT) littermates $(\mathrm{C} 3 \mathrm{H} / \mathrm{HeJ}$ and $\mathrm{C} 3 \mathrm{H} / \mathrm{OuJ}$ respectively, Jackson Laboratories, USA) were used $(n=3-5$ per group at day 7 and $\mathrm{n}=4-7$ per group at day 2). The co-dominant $\mathrm{LPS}^{\mathrm{d}}$ allele of $\mathrm{C} 3 \mathrm{H} / \mathrm{HeJ}$ mice was shown to correspond to a missense mutation in the third exon of the Toll-like receptor- 4 gene, thereby abolishing TLR 4 function ${ }^{12}$. The mice were injected i.p. four times at $2 \mathrm{~h}$ intervals with either $25 \mathrm{mg} / \mathrm{kg} \mathrm{MPTP}-\mathrm{HCl}$ or a corresponding volume of saline. This dose of MPTP resulted from preliminary dose-response results of MPTP intoxicated $\mathrm{C} 3 \mathrm{H} / \mathrm{OuJ}$ mice which showed an optimal dose of $25 \mathrm{mg} / \mathrm{kg}$ for these mice, known to be less susceptible to MPTP than C57/BL6 mice ${ }^{13}$. At day 2 and 7 for Iba-1 staining, at day 2 for MHC II staining or at day 7 for tyrosine hydroxylase (TH)staining and neurochemical analysis after MPTP intoxication, animals were processed for further analysis. MPTP metabolism was assessed and compared between WT and TLR4-deficient mice by injecting a single dose of $30 \mathrm{mg} / \mathrm{kg}$ MPTP$\mathrm{HCl}$. Animals were sacrificed $2 \mathrm{~h}$ and $4 \mathrm{~h}$ after this single injection.

Neurochemical analysis. Brains $(n=3-5$ per group) were rapidly removed and one side of the striatum was used for high-performance liquid chromatography (HPLC) measurement of DA and DA metabolites (3,4-dihydroxyphenylacetic acid (DOPAC), homovanillic acid (HVA) as previously described. In an independent set of experiments, the striatum was dissected for measurement of MPTP metabolism ${ }^{14,15}$. Tissue samples were weighted, transferred into $250 \mu \mathrm{l} 0.1 \mathrm{~N}$ perchloric acid contining $0.05 \%$ disodium EDTA and $0.05 \%$ sodium metabisulfite and sonicated for $10 \mathrm{sec}$. After centrifugation at $13,000 \mathrm{~g}$ at $4{ }^{\circ} \mathrm{C}$, the supernatants were passed through a $0.2 \mu \mathrm{m}$ filter (Spartan 13/0.2 RC, Schleicher \& Schuell, Germany) and collected for chromatographic assay.
The concentrations of DA and its metabolites (DOPAC and HVA) were determined by HPLC coupled with colometric detection. The detector potential was set at $750 \mathrm{mV}$ using a glassy carbon electrode and an $\mathrm{Ag} / \mathrm{AgCl}$ reference electrode. The detection limits were $1.5 \mathrm{pg}$ for DA and DOPAC and $50 \mathrm{pg}$ for $\mathrm{HVA}^{16}$. For $\mathrm{MPP}^{+}$measurement, striatal tissue was placed into $500 \mu \mathrm{l} 0.1 \mathrm{~N} \mathrm{HCLO} 4$ and were processed as described for catecholamines. $\mathrm{MPP}^{+}$was assessed by UV detection at a wavelength of $295 \mathrm{~nm}$. Detection limit was $30 \mathrm{pg}$.

Immunohistochemistry procedures in vivo. The posterior part of the brains were postfixed (4\% PFA), and cryoprotected. Immunohistochemistry was performed as described previously ${ }^{17}$ on free-floating cryomicrotome-cut sections $(20 \mu \mathrm{m})$ encompassing the entire midbrain. Sections were incubated with a polyclonal antibody against TH (1:1000; Pel-Freez Biologicals, Rogers, AR) and against ionized calcium-binding adaptor molecule 1 (Iba-1; WAKO, USA; $1: 500$ ), against MHC II as previously described ${ }^{18}$ (rat polyclonal anti-major histocompatibility complex class II, Serotec; $1 / 200$ ). Sections then were the treated with secondary antibodies (Vectastain, Vector Laboratories, Burlingame, CA) and subsequently incubated with avidin-biotinylated horseradish peroxidase complex. Peroxidase was revealed by incubation with $0.05 \% 3,3^{\prime}$-diaminobenzidine tetrahydrochloride containing $0.008 \%$ hydrogen peroxide, and sections were Nissl counter-stained.

Tyrosine hydroxylase $\mathrm{TH}+$ neurons revealed with the chromogen diaminobenzidine were analysed by bright field microscopy using a Nikon Optiphot- 2 microscope (Nikon France, Champigny/Marne, France) equipped with the Explora Nova VisioScan T4.18 software (La Rochelle, France) as previously described ${ }^{15}$. TH + cell bodies were quantified stereologically on regularly spaced sections covering the whole mesencephalon from the rostral pole of the substantia nigra to the locus coeruleus using the VisioScan stereology tool ${ }^{19}$. The investigator performing the quantification was blinded to the treatment groups during analysis. The total number of $\mathrm{SN} \mathrm{TH}^{+}$ neurons varied from 5860 to 8030 in saline treated WT and 7205 to 8070 in saline treated knock-outs, respectively. For microglia quantification, similarly adjacent (6-8) serial sections were used. An observer blind to the samples identity quantified the number of Ibal or MHC II-positive cells in the SN as described above.

Statistical analysis. Data are expressed as mean \pm S.E.M. Statistical analysis was performed using two-way ANOVA with genotype as one and saline/MPTP treatment as second independent variable, followed by post-hoc Student-Newman-Keuls test for all pairwise comparisons.

1. Fahn, S., Parkinsonism, P. S. \& Rowland, L. P. ed. Meritt's neurology 10th ed. New York: Lippincott, 2000, 679-693 (2000).

2. Hirsch, E. C. \& Hunot, S. Neuroinflammation in Parkinson's disease: a target for neuroprotection? Lancet Neurol 8, 382-397 (2009).

3. McGeer, P. L., Itagaki, S., Boyes, B. E. \& McGeer, E. G. Reactive microglia are positive for HLA-DR in the substantia nigra of Parkinson's and Alzheimer's disease brains. Neurology 38, 1285-1291 (1988).

4. Moresco, E. M., LaVine, D. \& Beutler, B. Toll-like receptors. Curr Biol 21, R488-493 (2011).

5. Laflamme, N. \& Rivest, S. Toll-like receptor 4: the missing link of the cerebral innate immune response triggered by circulating gram-negative bacterial cell wall components. Faseb J 15, 155-163 (2001).

6. Ros-Bernal, F. et al. Microglial glucocorticoid receptors play a pivotal role in regulating dopaminergic neurodegeneration in parkinsonism. Proc Natl Acad Sci U S A 108, 6632-6637 (2011).

7. Stefanova, N. et al. Toll-like receptor 4 promotes alpha-synuclein clearance and survival of nigral dopaminergic neurons. Am J Pathol 179, 954-963 (2011)

8. Beraud, D. \& Maguire-Zeiss, K. A. Misfolded alpha-synuclein and Toll-like receptors: therapeutic targets for Parkinson's disease. Parkinsonism Relat Disord 18 Suppl 1, S17-20 (2012).

9. Beraud, D. et al. alpha-Synuclein Alters Toll-Like Receptor Expression. Front Neurosci 5, 80 (2011).

10. Cote, M., Drouin-Ouellet, J., Cicchetti, F. \& Soulet, D. The critical role of the MyD88-dependent pathway in non-CNS MPTP-mediated toxicity. Brain Behav Immun 25, 1143-1152 (2011).

11. Drouin-Ouellet, J. et al. The role of the MYD88-dependent pathway in MPTP-induced brain dopaminergic degeneration. J Neuroinflammation 8, 137 (2011).

12. Poltorak, A. et al. Defective LPS signaling in $\mathrm{C} 3 \mathrm{H} / \mathrm{HeJ}$ and $\mathrm{C} 57 \mathrm{BL} / 10 \mathrm{ScCr}$ mice: mutations in Tlr4 gene. Science 282, 2085-2088 (1998).

13. Hamre, K., Tharp, R., Poon, K., Xiong, X. \& Smeyne, R. J. Differential strain susceptibility following 1-methyl-4-phenyl-1,2,3,6-tetrahydropyridine (MPTP) administration acts in an autosomal dominant fashion: quantitative analysis in seven strains of Mus musculus. Brain Res 828, 91-103 (1999).

14. Brochard, V. et al. Infiltration of CD4+ lymphocytes into the brain contributes to neurodegeneration in a mouse model of Parkinson disease. J Clin Invest 119, 182-192 (2009)

15. Alvarez-Fischer, D. et al. Modelling Parkinson-like neurodegeneration via osmotic minipump delivery of MPTP and probenecid. J Neurochem 107, 701-711 (2008).

16. Skrzydelski, D. et al. The chemokine stromal cell-derived factor-1/CXCL12 activates the nigrostriatal dopamine system. J Neurochem 102, 1175-1183 (2007). 
17. Hirsch, E., Graybiel, A. M. \& Agid, Y. A. Melanized dopaminergic neurons are differentially susceptible to degeneration in Parkinson's disease. Nature 334, 345-348 (1988).

18. Depboylu, C. et al. Upregulation of microglial Clq expression has no effects on nigrostriatal dopaminergic injury in the MPTP mouse model of Parkinson disease. J Neuroimmunol 236, 39-46 (2011).

19. Franklin, K. B. J. a. P. George The mouse brain in stereotaxic coordinates. Book (ISBN 0122660706 ) (1997)

20. Panaro, M. A. et al. Expression of TLR4 and CD14 in the Central Nervous System (CNS) in a MPTP Mouse Model of Parkinson's-Like Disease. Immunopharmacol Immunotoxicol 1-12 (2008).

21. Lin, J. J., Chen, C. H., Yueh, K. C., Chang, C. Y. \& Lin, S. Z. A CD14 monocyte receptor polymorphism and genetic susceptibility to Parkinson's disease for females. Parkinsonism Relat Disord 12, 9-13 (2006).

22. Hopkins, W. J. et al. Quantitative trait loci associated with susceptibility to bladder and kidney infections induced by Escherichia coli in female $\mathrm{C} 3 \mathrm{H} / \mathrm{HeJ}$ mice. J Infect Dis 199, 355-361 (2009).

23. Kanzler, H., Barrat, F. J., Hessel, E. M. \& Coffman, R. L. Therapeutic targeting of innate immunity with Toll-like receptor agonists and antagonists. Nat Med $\mathbf{1 3}$, 552-559 (2007).

24. Hennessy, E. J., Parker, A. E. \& O’Neill, L. A. Targeting Toll-like receptors: emerging therapeutics? Nat Rev Drug Discov 9, 293-307 (2010).

\section{Acknowledgements}

C.N. was supported by a postdoctoral grant from the Deutsche Forschungsgemeinschaft, (DFG), Germany. D.A.F. was supported by a postdoctoral grant from the Michael J. Fox Foundation (MJFF) and a research grant of the University Medical Center Giessen and
Marburg (UKGM). A.O. was supported by the Mildred-Scheel-Stiftung. T.L. was supported by a Master grant from the Fondation pour la Recherche Médicale (FRM). C.H. was supported by an M.D. thesis grant by the Boehringer Ingelheim Fond (BIF). E.C.H. and S.H. are investigators at the Centre National pour la Recherche Scientifique (CNRS). A.H. was supported by a "Poste Vert" (Accueil de Chercheurs Etrangers) from the Institut National de la Santé et de la Recherche Médicale (INSERM).

\section{Author contributions}

The work presented here was carried out in collaboration between all authors. C.N., S.H. and A.H. defined the research theme. C.N., S.H. and A.H. designed methods and experiments, carried out the laboratory experiments, analyzed the data, interpreted the results and wrote the paper. L.M., D.A.F., T.L., C.H., D.S., C.D. and L.L. co-designed experiments, discussed analyses, interpretation, and presentation. R.C.D., D.A.F. and E.C.H. analyzed the data and interpreted the results. All authors have contributed, seen and approved the manuscript. A.O., M.B. and P.P.M. co-worked on associated data collection and their interpretation.

\section{Additional information}

Supplementary information accompanies this paper at http://www.nature.com/ scientificreports

Competing financial interests: The authors declare no competing financial interests.

License: This work is licensed under a Creative Commons

Attribution-NonCommercial-NoDerivs 3.0 Unported License. To view a copy of this license, visit http://creativecommons.org/licenses/by-nc-nd/3.0/

How to cite this article: Noelker, C. et al. Toll like receptor 4 mediates cell death in a mouse MPTP model of Parkinson disease. Sci. Rep. 3, 1393; DOI:10.1038/srep01393 (2013). 